\title{
Functional nanocarriers by miniaturization of polymeric materials
}

\author{
"...the nano-world may be approached by miniaturization of \\ polymer materials transferring concepts realized in the bulk \\ to nanocarriers."
}

First draft submitted: 18 March 2016; Accepted for publication: 20 April 2016; Published online: 20 May 2016

Keywords: function $\bullet$ microscale $\bullet$ morphology $\bullet$ nanoscale $\bullet$ polymer

\section{Motivation \& strategies for building polymer-based nanocarriers}

The field of nanomedicine is aiming at a quantitative delivery of any type of drug selectively to a specific site of the body by aid of nanocarriers. Although this vision may not be realized in the near future, one has to appreciate the successful translation, for example, of polymer therapeutics like PEGylated cytokines toward blockbuster pharmaceutical products [1]. This example illustrates the impact that nanomedicine already has in healthcare. Aside from early speculations, there is continuously increasing evidence that size, shape and other physical cues of polymeric biomaterials are keys to cellular responses. For matrices larger than cells, the foreign body reaction to spheres from various materials depends on size [2]. For porous scaffolds or cavities infiltrated by cells, the topography of the materials like its structuring in nanofibers versus microfibers [3] or the local curvature of cavities [4] define cellular adhesion, cytoskeleton structure and intracellular signal transduction. In case of particulate carriers, it is well recognized that advantageous biological responses may arise for nanocarriers with sizes less than $1 \mu \mathrm{m}$, but also for systems with altered shapes, surface structures or incorporated compartments [5].

In principle, two main approaches toward nanocarriers may be distinguished. On the one hand, starting from the molecular level, single or few macromolecules of linear or grafted structure capable to build drug-poly- mer conjugates can be created. Polymers suitable for multivalent binding of a payload, for example, ionic interaction for condensation of nucleic acids in polyplexes, can form colloidal particles. The capabilities of these macromolecular carriers are largely determined by their chemical structure. In this way, direct physical interactions with biological systems (e.g., for targeting) or $\mathrm{pH}$-sensitive drug binding can be enabled.

On the other hand, the nano-world may be approached by miniaturization of polymer materials transferring concepts realized in the bulk to nanocarriers. Here, a fundamental principle is the organization and orientation of polymer chains in the polymer nanomatrix and at surfaces forming distinct phase morphologies. Polymer nanonetworks, in other words, solid colloidal particles, also belong to this group of nanomaterials, whereby the polymer chains are linked by covalent bonds.

It should be noted that the marketing authorization for nanomedicinal products, for example, by the European Medicines Agency (EMA) has predominantly been issued for systems from the first category, particularly for drug-polymer conjugates [6] . Does this mean that the second approach is not feasible to create functional nanocarriers?

\section{Functions of polymeric carriers base on structural principles on different hierarchical levels \\ The EMA has defined nanotechnology as "the use of tiny structures - less than 1000}
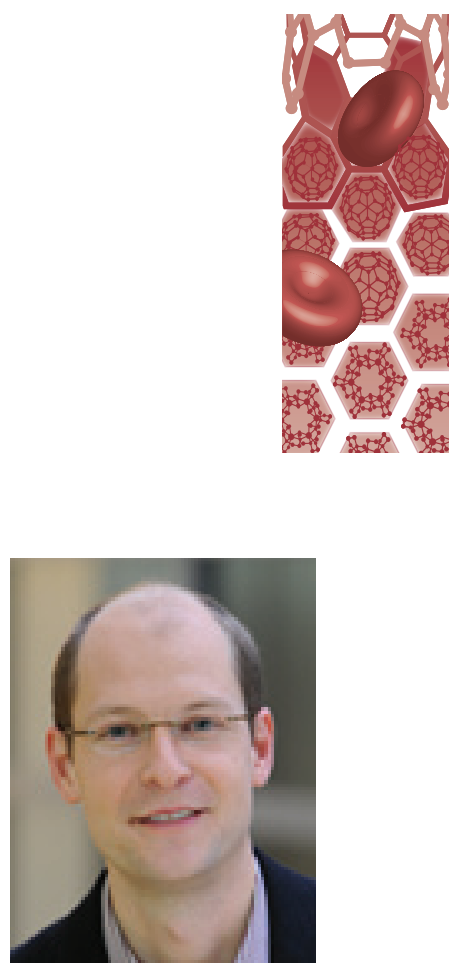

Christian Wischke

Institute of Biomaterial Science \& BerlinBrandenburg Centre for Regenerative

Therapies, Helmholtz-Zentrum Geesthacht, Kantstr. 55, 14513 Teltow, Germany

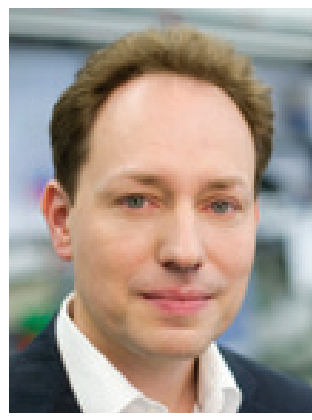

Andreas Lendlein

Author for correspondence: Institute of Biomaterial Science \& BerlinBrandenburg Centre for Regenerative Therapies, Helmholtz-Zentrum Geesthacht, Kantstr. 55, 14513 Teltow, Germany

and

Institute of Chemistry, University of Potsdam, Potsdam, Germany and

Department of Biology, Chemistry, \& Pharmacy, Freie Universität Berlin, Berlin, Germany

andreas.lendlein@hzg.de

Future $\because$ Medicine part of 
$\mathrm{nm}$ across - that are designed to have specific properties" [6]. The authors believe that 'functions' in addition to physical 'properties' should be the distinctive feature in nanomedicine.

A 'function' of a polymeric system can be defined as a specific capacity, which bases on distinct properties in combination with relevant physical processes, eventually leading to the desired application-relevant effect. The term function should here not be mistaken as reactive chemical group.

"...one can expect: functional nanocarriers can be realized by miniaturization of bulk materials, if the underlying structural principles are not severely affected."

A control of structures is essential to realize carrier functions. Here, 'structure' is not only restricted to the molecular level, but also includes the polymer morphology as well as larger structural features (e.g., compartments, pores, shape, etc.) [7]. Regarding the question raised above, one can expect: functional nanocarriers can be realized by miniaturization of bulk materials, if the underlying structural principles are not severely affected. Considering that distinct morphologies, for example, crystallites in semi-crystalline materials are in the low nanometer size range, they may be integrated in miniaturized carriers and contribute to their functions.

\section{Functions in carriers on the nanoscale: same concepts, same levels of control?}

The effect of size reduction on matrix-associated carrier function should here be exemplarily addressed for three cases: controlled drug delivery, degradation function and shape switching.

For carriers from hydrophobic polymers, the prominent concept to sustain drug release is a diffusion barrier, which may in principle be possible also for bulktype nanoparticles and nanonetworks. Substructures in the matrix such as crystalline domains or pores affect drug diffusion, which is furthermore influenced by concentration gradients, osmotic pumping mechanisms and/or, at least in the terminal release phase, by matrix polymer degradation. With decreasing particle sizes towards the nanometer range, the drug may not necessarily be embedded inside the matrix as desired, but partially or quantitatively adsorbed to the surface [8]. In case of successful incorporation, the reduced average diffusion length from the particle core to the surface may impact the efficiency of the polymer to act as diffusion barrier. Instead, distribution coefficients between polymer phase and dispersion medium may become increasingly relevant for release rates, if no additional retardation mechanisms (e.g., multivalent ionic interactions, specific binding motives, slowly cleavable drug-polymer bonds) are involved. While drug modification for retardation is academically very interesting, it may be less preferred for clinical translation based on regulatory hurdles. Therefore, physical concepts may better be followed, e.g., nanocapsules with rate-controlling membranes, which independent of the respective particle size may provide similar drug permeation at a given membrane thickness [9].

The degradation rate of matrices from copolyesters and other prominent examples of degradable materials depends on their susceptibility to hydrolysis as a process on the molecular level. While the same reaction mechanism applies, the altered length scales for diffusion of water and degradation products and the high surface-to-volume ratio can be very relevant for miniaturized matrices. The autocatalytic degradation in copolyesters by accumulated acidic degradation products is already strongly reduced when decreasing matrix size from the upper to the lower micrometersize range [10] - and may be missing for nanoparticles. The beneficial feature of some polymers to show surface erosion, which depends on their rapid hydrolysis and low water diffusion rates, will be lost with decreasing carrier size [11]. Bulk degrading materials with a relatively low rate of hydrolysis and low melting temperatures of degradation products to avoid crystallization may be feasible matrix materials for miniaturized particulate carriers.

The capacity of polymer particles to switch their shape by the shape-memory effect (SME) bases on a temporary storage of stress in the matrix and its release upon exposure to a suitable stimulus [12]. Those systems mechanistically distinguish from other concepts that partially translate a volume change by swelling into shape alteration [13]. The SME requires polymer network structures with permanent netpoints to define the shape of the relaxed state and an additional type of netpoints to fix a deformed, temporary shape. For shape-switchable particles from thermoplastic multiblock copolymers, networks with physical netpoints from two types of crystalline domains were employed, forming the permanent and temporary netpoints, respectively [12]. As each of these crystalline domains has spatial requirements, a reduction of matrix size may lead to a loss of SME as supported by systematically decreasing degrees of crystallinity for nanoparticles [12]. Still, alternative structural concepts may be applied. One approach may be to replace crystalline domains that form the permanent netpoints by covalent junctions [14]. Another one may be to use, for example, photocleavable links as temporary netpoints [15]. With those measures, a shape switching function by the SME might be possible for nanoparticles. 


\section{Future perspective}

The prediction and control of biological functions of nanocarriers remains an important goal for present research. While there is an increasing recognition that many preclinical models may need to be challenged for their predictive power, a critical review should also be applied to structural principles associated with carrier functions in the context of a matrix size confinement. In some cases, alternative structural approaches may be needed to preserve carrier functionality upon miniaturization from micro- to nanocarriers. This may also include polymer nanonetwork particles, for which a major challenge will be to create very well-defined molecular architectures.

While submicron particles can be accessible by modified dispersion techniques, it remains an important task to routinely obtain very small bulk-type nanoparticles by a miniaturization approach. Furthermore, creating nanoparticles with defined phase morphologies is challenging. Additionally, alternative matrix materials should be explored in more

\section{References}

1 Duncan R. Polymer therapeutics: top 10 selling pharmaceuticals - what next? J. Control. Release 190, 371-380 (2014).

2 Veiseh O, Doloff JC, Ma ML et al. Size- and shapedependent foreign body immune response to materials implanted in rodents and non-human primates. Nat. Mater. 14(6), 643-651 (2015).

3 Stevens MM, George JH. Exploring and engineering the cell surface interface. Science 310(5751), 1135-1138 (2005).

4 Xu X, Wang W, Kratz K et al. Controlling major cellular processes of human mesenchymal stem cells using microwell structures. Adv. Healthc. Mater. 3(12), 1991-2003 (2014).

5 Mitragotri S, Lahann J. Physical approaches to biomaterial design. Nat. Mater. 8(1), 15-23 (2009).

6 Noorlander CW, Kooi MW, Oomen AG, Park MVDZ, Vandebriel RJ, Geertsma RE. Horizon scan of nanomedicinal products. Nanomedicine 10(10), 1599-1608 (2015).

7 Behl M, Razzaq MY, Lendlein A. Multifunctional shapememory polymers. Adv. Mater. 22(31), 3388-3410 (2010).

8 Francis D, Mouftah S, Steffen R, Beduneau A, Pellequer Y, Lamprecht A. Ion milling coupled field emission scanning electron microscopy reveals current misunderstanding of detail, for example, polydepsipeptides with modified degradation pattern. Adapted analytical methods, particularly high-resolution imaging methods for dynamic systems will be important to illustrate how structural elements contribute to nanocarrier functions. Overall, multifunctional nanoparticles from miniaturized polymeric materials, which combine several functions established for bulk materials, may contribute to new therapeutic approaches for nanomedicine.

\section{Financial \& competing interests disclosure}

This work is associated to the authors' efforts to design new nanocarriers within the Collaborative Research Center 1112 (project A03) funded by the German Research Council. The authors have no other relevant affiliations or financial involvement with any organization or entity with a financial interest in or financial conflict with the subject matter or materials discussed in the manuscript apart from those disclosed.

No writing assistance was utilized in the production of this manuscript.

morphology of polymeric nanoparticles. Eur. J. Pharm. Biopharm. 89, 56-61 (2015).

9 Musyanovych A, Landfester K. Polymer micro- and nanocapsules as biological carriers with multifunctional properties. Macromol. Biosci. 14(4), 458-477 (2014).

10 Li L, Schwendeman SP. Mapping neutral microclimate $\mathrm{pH}$ in PLGA microspheres. J. Control. Release 101(1-3), 163-173 (2005).

11 Von Burkersroda F, Schedl L, Gopferich A. Why degradable polymers undergo surface erosion or bulk erosion. Biomaterials 23(21), 4221-4231 (2002).

12 Wischke C, Schossig M, Lendlein A. Shape-memory effect of micro-/nanoparticles from thermoplastic multiblock copolymers. Small 10(1), 83-87 (2014).

13 Klinger D, Wang CX, Connal LA et al. A facile synthesis of dynamic, shape-changing polymer particles. Angew. Chem. Int. Edit. 53(27), 7018-7022 (2014).

14 Friess F, Nochel U, Lendlein A, Wischke C. Polymer micronetworks with shape-memory as future platform to explore shape-dependent biological effects. Adv. Healthc. Mater. 3(12), 1986-1990 (2014).

15 Jiang HY, Kelch S, Lendlein A. Polymers move in response to light. Adv. Mater. 18(11), 1471-1475 (2006). 